\title{
Un passage de l'Évangile selon Matthieu illustré: O.Moen inv. 631
}

Édité par P.J. Sijpesteijn en 1984, l'O.Moen inv. $631\left(\mathrm{MP}^{3} 2699.5=\right.$ LDAB $2954=$ TM 61800) mesure 10,4 cm de large sur $11,5 \mathrm{~cm}$ de haut $\left({ }^{1}\right)$. Il est brisé dans sa partie supérieure et sur le côté droit. De provenance inconnue et actuellement non localisé $\left({ }^{2}\right)$, il a été daté paléographiquement par son éditeur des $\mathrm{V}^{\mathrm{e}} / \mathrm{VI}^{\mathrm{e}}$ siècles. Si le côté concave de l'ostracon, de couleur rougeâtre, est vierge, l'autre face porte quatre dessins décrits par P.J. Sijpesteijn comme deux représentations d'un petit homme et deux d'un oiseau, que surmontent quatre lignes de texte identifiées comme l'Évangile selon Matthieu, 1, 19-20, qui auraient été copiées en contexte scolaire. Voici le texte, légèrement retouché, de l'édition $\left(^{3}\right)$ :

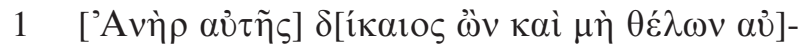

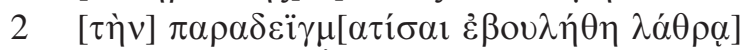

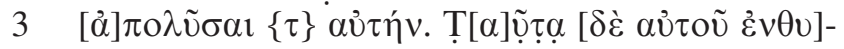

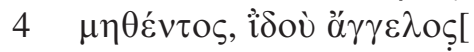

(*) Dans cet article, les abréviations utilisées sont celles de l'Année Philologique, à compléter par DACL (= Dictionnaire d'Archéologie Chrétienne et de Liturgie), DPAC (= Dizionario Patristico e di Antichità Cristiane), NDPAC (= Nuovo Dizionario Patristico $e$ di Antichità Cristiane) et RAC (= Reallexikon für Antike und Christentum). Cet article a été rédigé durant notre séjour de recherches à l'Academia Belgica de Rome, effectué du $1^{\text {er }}$ mars au 31 mai 2012, grâce à un Stipendium de cette institution. Nous remercions vivement Madame M.-H. Marganne, notre directrice de thèse, pour nous avoir fait part de ses remarques et de ses conseils, ainsi que Monsieur M. Mélard, pour ses informations bibliographiques. Nos plus vifs remerciements s'adressent également à Monsieur A. Delattre, à qui nous sommes redevable de plusieurs améliorations et suggestions, ainsi qu'au «referee» anonyme.

(1) P.J. SiJPESTEIJN, «Matthäus 1,19-20 auf einem Ostrakon», ZPE 55 (1984), p. 145 et pl. II k.

(2) Information confirmée par K.A. Worp (message électronique du 30.10.2011) et par D. Martinez (messages électroniques des 05.04.2012 et 08.05.2012), que nous remercions.

(3) Les modifications au texte édité par P.J. Sijpesteijn portent sur 1) 'Avì $\rho$ (ligne 1), dont il restituait le $\mathrm{A}$ initial sur une ligne précédente, 2) sur $\lambda \alpha \dot{\alpha} \theta \rho \alpha$ (ligne 2), au lieu de

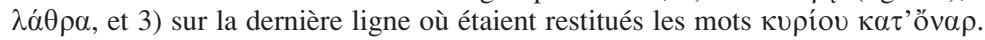

Chronique d'Égypte LXXXVII (2012), fasc. 174 - doi: 10.1484/J.CDE.1.103138 
«Son mari, qui était un homme juste et qui ne souhaitait pas la diffamer, voulut la répudier en cachette $\left({ }^{4}\right)$. Tandis qu'il y réfléchissait, voici qu'un ange (lacune)».

Ce passage de l'Évangile selon Matthieu est également attesté dans trois autres témoins papyrologiques, à savoir P.Mich. inv. $4942+$ P.Freib. inv. 615 + P.Pierpont Morgan inv. M 615 (van Haelst $324+350=$ LDAB 2925 = TM 61773) $\left({ }^{5}\right)$, évangéliaire bilingue gréco-copte sur parchemin daté des $\mathrm{VII} / \mathrm{VIII}{ }^{\mathrm{e}}$ siècles par $\mathrm{A}$. Boud'hors $\left({ }^{6}\right)$ et provenant peut-être du Monastère Blanc $\left({ }^{7}\right)$, P.Oxy. 1.2 (P.Univ.Pennsylv.Mus. inv. E 2746 = van Haelst $332=\mathfrak{P}^{1}=$ LDAB $2940=$ TM 61787), fragment de codex daté du III $^{\mathrm{e}}$ siècle et contenant des extraits des deux premiers chapitres de l'Évangile selon Matthieu, et, enfin, le Codex Sinaïticus $($ LDAB $3478=$ TM 62315), daté de 360 de notre ère environ $\left({ }^{8}\right)$. Ainsi que l'avait remarqué P.J. Sijpesteijn, si O.Moen inv. 631, comme le Codex Sinaïticus, atteste le verbe composé $\pi \alpha \rho \alpha \delta \varepsilon \imath \gamma \mu \alpha \tau i ́ \sigma \alpha 1$, «frapper d'un blâme, d'une peine qui serve d'exemple, rendre infâme, déshonorer», P.Oxy. 1.2, qui est le plus ancien témoin, porte la forme simple $\delta \varepsilon \imath \gamma \mu \alpha \tau i ́ \sigma \alpha 1$, «faire un exemple», leçon adoptée par l'édition Nestle-Aland $\left({ }^{9}\right)$. Quant à l'évangéliaire bilingue, non répertorié par P.J. Sijpesteijn dans les passages parallèles, il atteste, lui aussi, $\pi \alpha \rho \alpha \delta \imath \gamma \mu \alpha \tau i ́ \sigma \alpha$, avec iotacisme de la diphtongue $\varepsilon 1$.

Contrairement au texte, les illustrations de cet ostracon n'ont jamais fait l'objet d'une étude approfondie. P.J. Sijpesteijn, suivi par K. Treu $\left({ }^{10}\right)$, les

(4) Selon U. HolzmeISTER, De sancto Ioseph quaestiones biblicae (= Scripta Pontificii

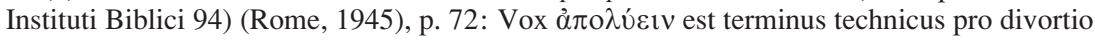
( «e mot $\alpha \dot{\tau} \mathrm{o} \lambda \hat{\varepsilon} \varepsilon v$ est le terme technique pour divorce»). Pour le verset 19, voir aussi la traduction que donne X. LÉON-Dufour, «L'annonce à Joseph», dans Mélanges bibliques rédigés en l'honneur de André Robert (Paris, 1957), p. 390.

(5) Pour une présentation générale du codex, voir K. SchüssLER (éd.), Biblia Coptica. Die koptischen Bibeltexte, III: Das sahidische Alte und Neue Testament, 4: sa 561-585 (Wiesbaden, 2006), pp. 46-64. Le passage de l'Évangile selon Matthieu, 1, 19-20 se trouve au folio $23^{\mathrm{v}}$, dans la partie du codex conservée à la Pierpont Morgan Library de New York. Pour un fac-similé du manuscrit, voir H. Hyvernat, Bybliothecae Pierpont Morgan Codices Coptici Photografice expressi, XI: Codex M 615. Evangeliarium Graeco-Sahidice (Rome, 1922).

(6) A. Boud'HORs, «Toujours honneur au grec? À propos d'un papyrus gréco-copte de la région thébaine», dans A. PAPACONSTANTinou (éd.), The Multilingual Experience in Egypt from the Ptolemies to the Abassids (Burlington, 2010), p. 187.

(7) J. VAn HaElst, Catalogue des papyrus littéraires juifs et chrétiens (Paris, 1976), p. $121, \mathrm{n}^{\circ} 324$.

(8) Une reproduction intégrale et de haute qualité du codex, accompagnée d'une transcription diplomatique, est accessible à l'adresse www.codexsinaiticus.org (dernière consultation le 19.05.2012).

(9) E. Nestle \& K. Aland (éd.), Novum Testamentum Graece (Stuttgart, 200727).

(10) K. Treu, «Christliche Papyri XII», APF 32 (1986), p. 88, n 332a. 
qualifiait de «dessins primitifs» (primitiven Zeichnungen) de deux hommes et, tête-bêche, de deux oiseaux. Dans son ouvrage sur les papyrus, les parchemins et les papiers illustrés, U. Horak les décrivait plutôt comme des esquisses (Skizzen) $\left({ }^{11}\right)$ et acceptait l'identification de P.J. Sijpesteijn de deux figures humaines et de deux oiseaux.

L'étude de la forme, de la «mise en page» et du contenu d'O.Moen inv. 631 dans le cadre de nos recherches sur les papyrus scolaires grecs et latins chrétiens $\left({ }^{12}\right)$, nous a permis de proposer une nouvelle identification de la scène réunissant les deux personnages, en rapport avec le texte de l'Évangile. Les quatre lignes écrites sur l'ostracon relatent l'épisode du premier songe de Joseph, durant lequel l'ange du Seigneur lui annonce la grossesse miraculeuse de Marie. Le même évangile rapporte deux autres manifestations de l'ange à Joseph, pour lui conseiller de fuir en Égypte (Év. Matt., 2, 13), puis, durant le séjour en Égypte, pour lui annoncer qu'il peut rentrer en Palestine (Év. Matt., 2, 19-20). On trouve des récits plus détaillés du premier songe de Joseph dans la littérature apocryphe chrétienne, par exemple, dans le Protévangile de Jacques, XIII-XIV $\left({ }^{13}\right)$, et dans l'Histoire de Joseph le charpentier, V-VI $\left({ }^{14}\right)$. Dans ce dernier texte, l'ange est identifié avec l'archange Gabriel.

(11) U. HoraK, Illuminierte Papyri, Pergamente und Papiere, I (= Pegasus Oriens, 1) (Vienne, 1992), p. 256, n 325.

(12) N. CARLIG, Les papyrus scolaires grecs et latins chrétiens, mémoire de maîtrise, Université de Liège, 2011, pp. 139-140. Depuis novembre 2011, notre catalogue des papyrus scolaires grecs et latins chrétiens, qui comprend 104 exercices scolaires, dont 24 sur ostraca, est accessible, sur le site web du CEDOPAL, à l'adresse <http://promethee.philo. ulg.ac.be/cedopal/Bibliographies/Scolaires_chretiens_nov2011.htm>, avec une bibliographie (http://promethee.philo.ulg.ac.be/cedopal/Bibliographies/Bibl_papyrus\%20scolaires\% 20grecs\%20 et\%20latins\%20chretiens_nov2011.htm).

(13) Si l'édition de référence est celle d'É. DE STRYCKER (éd.), La forme la plus ancienne du Protévangile de Jacques. Recherches sur le Papyrus Bodmer 5 avec une édition critique du texte grec et une traduction annotée (= Subsidia hagiographica, 33) (Bruxelles, 1961), on ne manquera pas de consulter également l'édition récente d'un fragment de codex de papyrus d'Oxford par J. BINGEN, «Protévangile de Jacques, XIII-XV (P. Ashmolean inv. 9)», $C E 80$ (2005), pp. 201-214, qui transmet des leçons particulières. Le texte du Protévangile de Jacques nous est connu également par des versions en syriaque, arménien, éthiopien et vieux-slave. Voir [D. DoRÉ], «Apocryphes du N. T.», Dictionnaire encyclopédique de la Bible, $3^{\mathrm{e}}$ éd. (Turnhout, 2002) pp. 120-127, spéc. p. 122: «25. Jacques, Protévangile».

(14) P. PeEters (trad.), «Histoire de Joseph le charpentier», dans C. Michel \& P. PeEters (éd.), Évangiles apocryphes (= Textes et documents, 13) (Paris, 1911) pp. 191245, qui contient la traduction française des rédactions copte (bohaïrique) et arabe. Outre ces versions, on en possède également une en latin. L'original grec est perdu. Voir [D. DoRÉ], «Apocryphes du N. T.», Dictionnaire encyclopédique de la Bible, $3^{\mathrm{e}}$ éd. (Turnhout, 2002) pp. 120-127, spéc. p. 123: «30. Joseph le charpentier, Histoire». 
Les quatre illustrations se trouvent immédiatement au-dessous du texte. Si les deux figures animales, dessinées tête-bêche par une seconde main, n'ont apparemment rien à voir avec le texte, il nous semble en revanche que les deux personnages peuvent être mis en relation avec l'épisode du songe de Joseph copié par l'élève. Dans le sens du texte et dans la partie inférieure de l'ostracon, on voit en effet deux représentations humaines, qui ont toutes deux les bras levés, et qui sont en partie endommagées par une tache noire, comme si on avait tenté de les effacer. Les corps, têtes comprises, sont sommairement tracés au moyen de traits droits formant des rectangles auxquels sont attachés des bras sous forme de simples traits se prolongeant par cinq autres petits traits représentant les doigts. Le bras droit du personnage situé dans le coin inférieur gauche de l'ostracon suit la cassure du support, qui semble donc être d'origine de ce côté. Le personnage pourrait être agenouillé ou assis: on devine en effet un trait partant à angle droit du bas du corps, vers la droite, qui fait penser à une jambe pliée. Il ne nous semble pas être un orant, qui est habituellement représenté, non pas avec les bras simplement levés, mais bien écartés, et toujours debout $\left({ }^{15}\right)$. Quoiqu'il ne soit pas couché, mais peut-être appuyé ou assis le long de la cassure, il pourrait représenter Joseph, qui a pu se voir lui-même en rêve, ou qui a été réveillé par l'apparition de l'ange $\left({ }^{16}\right)$. Les bras levés et tendus pourraient traduire sa stupéfaction, voire sa peur face à l'arrivée de l'ange. Il faut aussi tenir compte de la maladresse du dessinateur, qui se manifeste également dans l'écriture peu soignée $\left({ }^{17}\right)$, du type de support, exigu et non plan, sur lequel il n'est pas aisé d'écrire et, a fortiori, de dessiner, et, enfin, de la liberté qu'a prise l'élève pour exécuter son croquis. Quant au personnage dessiné plus haut, à droite, nous proposons d'y voir l'ange et d'interpréter le dessin comme celui du premier songe de Joseph, en lien direct avec le texte de l'Évangile. En effet, sous les taches résultant probablement d'une tentative de gommage, on distingue les formes de deux ailes qui partent du dessus de la tête et se

(15) H. LECLERCQ, «Orant», DACL XII, 2 (1936), coll. 2291-2322, spéc. coll. 22912299: «I. Signification du geste» où est notamment cité TERTULLIEN, De Oratione, 14; «II Le geste païen»; «III. Le geste chrétien» et 2312-2314: «VIII. Art copte», et T. KLAUSER, «Geste und Gebärde», RAC X (1978), coll. 895-902, spéc. «A. III. Hand/Finger».

(16) Nous n'aborderons pas ici la question débattue depuis longtemps de savoir si l'ange est apparu à Joseph en songe ou lorsqu'il était à l'état de veille. À ce sujet, voir, par exemple, J. Knabenbauer, Commentarius in quatuor S. Evangelia (Paris, 1892), pp. 59-60.

(17) Selon la typologie des mains d'élèves établies par R. CRIBIORE, Writing, Teachers and Students in Graeco-Roman Egypt (= ASP, 36) (Atlanta, 1996), pp. 102-118, nous classerions celle d'O.Moen inv. 631 dans la $3^{\mathrm{e}}$ catégorie, dénommée «evolving». 
séparent en deux traits horizontaux ondulant légèrement. Si les conditions de conservation de l'ostracon et les taches noires ne permettent pas de distinguer clairement un nimbe $\left({ }^{18}\right)$, on pourrait éventuellement en deviner des traces entre les deux ailes et au-dessus de celles-ci.

Dans les papyrus, on trouve six autres représentations d'ange. Un premier exemple est attesté sur le coupon de papyrus PSI 15.1571 (MP3 2916.6 = LDAB 10142 = TM 68872), provenant d'Antinoé et daté, d'après son premier éditeur, du $\mathrm{VI}^{\mathrm{e}}$ siècle $\left({ }^{19}\right)$. Le fragment contient la moitié gauche de la tête nimbée et du torse de l'ange. Une autre représentation d'ange est dessinée au verso $(\downarrow)$ de P.NYU inv. 546 (LDAB $10199=$ TM 68929) $\left({ }^{20}\right)$, daté du VI $\mathrm{I}^{\mathrm{e}}$ siècle, tandis que le recto $(\rightarrow)$ contient une représentation humaine qualifiée de «magique» $\left({ }^{21}\right)$. On distingue notamment un trait vertical qui part de l'épaule de l'ange et retombe en un trait oblique ondulé, que l'éditrice du papyrus interprète comme une aile $\left({ }^{22}\right)$. Un troisième ange est dessiné dans la Chronique alexandrine illustrée (P.Goleniscev inv. $310+$ P.Vindob. inv. K $11630=$ van Haelst $631=$ MP $^{3}$ 2244 = LDAB 6345 = TM 65104), datée du VI e siècle par G. Cavallo et H. Maehler $\left.{ }^{23}\right)$. Celui-ci se trouve en compagnie d'autres personnages dans la partie droite du fragment. Dessiné en couleurs, il peut être identifié à coup sûr, car il apparaît nimbé et pourvu d'ailes dorsales dont on distingue la partie supérieure, remontant à hauteur du cou $\left({ }^{24}\right)$. Enfin, bien que s'éloignant du type de l'ange nimbé et ailé tel que représenté dans les papyrus évoqués jusqu'ici, trois représentations d'anges, toutes sur parchemin et dont le contexte est considéré comme magique, sont également attestées dans les papyrus magiques P.Lond.Copt. 1.524 (Brit.Libr. inv. Or. 5525), non daté (LDAB 98056 = TM 98056), P.Heid. inv. K 1681,

(18) Pour H. LeCLERCQ, «Nimbe», DACL XII, 1 (1935), col. 1302, «l'ange du Seigneur se montre nimbé au $\mathrm{V}^{\mathrm{e}}$ siècle». Par ailleurs, il observe que l'ange représenté sur un ivoire du British Museum (voir ID., «Anges», DACL I, 1 [1907], col. 2109, fig. 627) daté du IV ${ }^{\mathrm{e}}$ siècle, n'est pas nimbé.

(19) S. SeTTIS, «Papiri figurati», ASNP, $2^{\text {e }}$ sér., 35 (1966), pp. 22-25 et pl. IV.

(20) Édité par HORAK, op. cit., pp. 155-157.

(21) HORAK, op. cit., p. 156.

(22) HORAK, op . cit., p. 155.

(23) G. Cavallo \& H. Maehler, Greek Bookhands of the Early Byzantine Period: A.D. 300-800 (= BICS Suppl., 47) (Londres, 1987), p. 82.

(24) A. BAuer \& J. STRZYGOWSKI, «Eine Alexandrinische Weltchronik», Denkschriften der kaiserlichen Akademie der Wissenschaften. Philosophisch-Historische Klasse 51 (1906), p. 163 et pl. VII recto D. 
daté du $X^{\mathrm{e}}$ siècle $($ LDAB $99609=$ TM 99609), et P.Heid. inv. K 518, daté entre le IX ${ }^{\mathrm{e}}$ et le $\mathrm{XI}^{\mathrm{e}}$ siècle (TM 99553) $\left({ }^{25}\right)$.

Dans l'art copte, particulièrement dans les peintures murales, on trouve de nombreuses représentations d'anges. Ainsi, on peut voir l'archange Gabriel, nimbé et ailé, au côté de la Vierge Marie, dans une représentation de l'Annonciation, peinte dans le monastère de Baouit (Chapelle LI) ${ }^{26}$ ). À la suite des fouilles récentes, il est admis que les peintures de Baouit datent en général des $\mathrm{VI}^{\mathrm{e}}-\mathrm{VIII}^{\mathrm{e}}$ siècles. Datée du $\mathrm{VIII}^{\mathrm{e}}$ siècle par $\mathrm{K}$. Innemée $\left({ }^{27}\right)$, la peinture de l'Annonciation de la conque ouest de l'abside du monastère des Syriens (Deir al-Sourian) fournit un autre exemple. Faisant partie d'un programme iconographique comprenant aussi une Épiphanie, la scène qui nous intéresse ici, représente la Vierge et l'archange Gabriel, ailé et nimbé, entourés des prophètes Moïse, Isaïe, Ézéchiel et Daniel, avec, en arrière-fond, la ville de Nazareth. Datée du $\mathrm{VI}^{\mathrm{e}}$ siècle, la scène du sanctuaire rupestre d'Abou Hennis, près d'Antinoé, représentant Joseph couché visité par un ange, fait référence au deuxième songe, annonçant la fuite en Égypte, qui est ensuite représentée sur la paroi $\left({ }^{28}\right)$.

(25) U. HoraK, «Christliches und Christlich-Magisches auf illuminierten Papyri, Pergamenten, Papieren und Ostraka», MiChA 1 (1995), pp. 27-48, spéc. pp. 41-43: «4. Engel». L'auteur commente également les anges de PSI 15.1571 et de P.NYU inv. 546.

(26) J. ClÉDAT, Le monastère et la nécropole de Baouit, notes mises en œuvre et éditées par D. BÉnAzeth et M.-H. Rutschowscaya (= MIFAO, 111) (Le Caire, 1999), p. 113 (description) et p. 127 et fig. 109. Cette peinture était datée par J. Clédat d'abord du VIII ${ }^{\mathrm{e}}$ ou $\mathrm{IX}^{\mathrm{e}}$ siècle (J. ClÉDAT, op. cit., p. 110), puis rétrodatée «dans le courant du $\mathrm{VI}^{\mathrm{e}} \mathrm{s}$. ou commencement du VII ${ }^{\mathrm{e}}$ (J. ClÉDAT, op. cit., p. 110, n. 63).

(27) Voir K.C. InNEMÉE, «A Newly Discovered Painting of the Epiphany in Deir alSurian», Hugoye 14, 1 (2011), pp. 63-85. C'est sous une Ascension du XIII ${ }^{\mathrm{e}}$ siècle que fut découverte cette Annonciation. Pour une description de la peinture, voir P. LAFERrière, $L a$ Bible murale dans les sanctuaires coptes $(=$ MIFAO, 127) (Le Caire, 2008), pp. 58-65, spéc. pp. 63-65 et pl. XVII-XVIII: «L'Annonciation de la conque ouest». De nombreux travaux préliminaires relatifs à la découverte, à la restauration et à la datation des peintures du Deir al-Sourian ont été publiés dans la revue Hugoye. Journal of Syriac Studies. On consultera, par exemple, K.C. INNEMÉE, «Recent Discoveries of Wall-Paintings in Deir AlSurian», Hugoye 1, 2 (1998), pp. 288-304; ID., «Deir Al-Surian (Egypt): its Wall-Paintings, Wall-Texts, and Manuscripts, I: The Wall-Paintings of Deir Al-Surian: New Discoveries of 1999», Hugoye 2, 2 (1999), pp. 167-188 et ID., «Deir Al-Surian (Egypt): Conservation Work. Autumn 2000», Hugoye 4, 2 (2001), pp. 259-268, spéc. 258-265. Dans ce vaste projet, la datation des peintures est toujours sujette à modification.

(28) J. CLÉDAT, «Notes archéologiques et philologiques», BIFAO 2 (1902), pp. 41-70, spéc. p. 50 et pl. II; H. LECLERCQ, «Âne», DACL I, 1 (1907), coll. 2057-2058 (fig. 599); ID., «Joseph (Saint)», DACL VII, 2 (1927), col. 2663; T. KLAUSER, «Engel X (in der Kunst)», RAC V (1962), col. 289 (n $\left.\mathrm{n}^{\circ} 134 \mathrm{~b}\right)$ et 295 ( $\left.\mathrm{n}^{\circ} 15\right)$; M. MARINONE, «Giuseppe (san). II. Iconografia», DPAC II (1984), col. 1619, et NDPAC II (2007), col. 2326. Voir aussi M. ZIBAWI, Images de l'Égypte chrétienne. Iconologie copte (Paris, 2003), pp. 58-66, spéc. p. 59 et fig. 57 et G.J.M. VAn Loon \& A. Delattre, «Le cycle de l'enfance du Christ 
Malgré son mauvais état de conservation, on distingue cependant nettement la partie supérieure des ailes et le nimbe de l'ange visitant Joseph, qui est couché. Une autre représentation du premier songe de Joseph a été découverte, en 2006, à Baouit sur le mur nord de la salle S7 $\left({ }^{29}\right)$.

En Occident, on trouve des représentations du songe de Joseph dès la fin du IV e siècle, avec le sarcophage du Puy, où l'ange apparaît nimbé, mais dépourvu d'ailes $\left({ }^{30}\right)$. Datées de 435 , sous le pontificat de Sixte III, la bande supérieure gauche des mosaïques de l'arc triomphal de la basilique Sainte-Marie-Majeure à Rome $\left({ }^{31}\right)$ représente une Annonciation, qui contient une adaptation du premier songe de Joseph, où l'on voit celui-ci en conversation avec deux anges, une Présentation au Temple et, peutêtre, une représentation du deuxième songe de Joseph, relatif à la fuite en Égypte ${ }^{32}$ ). Les nombreux anges de ces mosaïques sont tous nimbés et ailés. On répertorie enfin des représentations du premier songe de Joseph sur une boîte en ivoire provenant sans doute de l'abbaye bénédictine de Werden (Westphalie, Allemagne), datée d'environ $800\left({ }^{33}\right)$ et sur les

dans l'église rupestre de Saint-Jean-Baptiste à Deir Abou Hennis», dans A. Boud'HoRs, J. Gascou \& D. Vaillancourt (éd.), Études coptes IX. Onzième journée d'études (Strasbourg, 12-14 juin 2003) (= Cahiers de la Bibliothèque copte, 14) (Paris, 2006), pp. 119134, spéc. p. 126 et fig. 6-7.

(29) M.-H. Rutschowscaya, «Nouvelles peintures découvertes dans le monastère de Baouit», dans A. Boud'Hors \& C. LouIs (éd.), Études coptes XI. Treizième journée d'études (Marseille, 7-9 juin 2007) (= Cahiers de la Bibliothèque copte, 17) (Paris, 2010), pp. 45-51, spéc. p. 46 et pl. 2, fig. B.

(30) H. LECLERCQ, «Joseph (Saint)», DACL VII, 2 (1927), coll. 2662-2663 et M. MARINONE, «Giuseppe (san). II. Iconografia», DPAC II (1984), col. 1619 et NDPAC II (2007), col. 2326.

(31) H. LECLERCQ, «Ange», DACL I, 1 (1907), col. 2110 et fig. 625; ID., «Joseph (Saint)», DACL VII, 2 (1927), col. 2663 et M. MARINONE, «Giuseppe (san). II. Iconografia», DPAC II (1984), col. 1619 et NDPAC II (2007), col. 2326. Pour T. KLAUSER, op. cit., col. $265\left(n^{\circ} 32 \mathrm{~b} 2\right)$ et $295\left(n^{\circ} 15\right)$, il ne s'agit pas du songe de Joseph. Sur la basilique et ses mosaïques, voir la présentation générale dans M.R. MiLES, «Santa Maria Maggiore's FifthCentury Mosaics: Triumphal Christianity and the Jews», HThR 86 (1993), pp. 155-175, spéc. pp. 157-160. Sur les propositions d'interprétation des mosaïques, outre l'article de M.R. Miles ci-dessus, se référant à une annonce à Joseph (p. 159), voir aussi A. GEYER, «Bibelepik und frühchristliche Bildzyklen: die Mosaiken von Santa Maria Maggiore in Rom», MDAI(R) 112 (2005-2006), pp. 293-321, qui parle également d' «annonce de l'ange à Joseph portant un bâton» (p. 302) et J. MARTIN, Der Weg zur Ewigkeit führt über Rom: Die Frühgeschichte des Papstums und die Darstellung der neutestamentlichen Heilsgeschichte im Triumphbigenmosaik von Santa Maria Maggiore in Rom (Stuttgart, 2010).

(32) Pour M. MARINONE, «Giuseppe (san). II. Iconografia», DPAC II (1984), col. 1620, et NDPAC II (2007), col. 2326, cette identification reste hypothétique à cause de l'état de délabrement de la mosaïque.

(33) Aujourd'hui conservés au Victoria \& Albert Museum de Londres (inv. 149-1866, 149a-1866 et 149b-1866), les 3 panneaux ont été incisés probablement à partir d'un diptyque tardo-antique réutilisé à l'époque carolingienne. Voir W.F. VolBACH, Elfenbeinarbeiten 
plaques d'ivoire de la Chaire de Maximien de Ravenne $\left({ }^{34}\right)$, datée d'environ $547\left({ }^{35}\right)$.

Tête-bêche par rapport au texte et aux figures humaines, les deux autres dessins de l'ostracon O.Moen inv. 631 représenteraient, d'après P.J. Sijpesteijn, suivi par K. Treu et U. Horak, des oiseaux, dont l'un est moins visible à cause d'une tache noire. Vu l'épaisseur du trait, leur position tête-bêche par rapport au reste du contenu et leur empiètement sur le texte, ils ont certainement été exécutés par une main postérieure à celle qui a copié le texte et dessiné Joseph et l'ange. L'identification de ces deux figures animales à des oiseaux est fondée sur la présence de becs et d'ailes partant de leur partie postérieure. Le premier aurait été effacé par le scripteur, ce qui expliquerait la tache noire qui le cache partiellement, et le second aurait été réalisé suite à cette opération. On ne peut cependant pas expliquer la présence de trois pattes pour le second oiseau. Les deux premières étaient-elles dessinées trop en avant du corps et le scripteur a-til décidé d'en redessiner à une position plus centrale? Dans ce cas, pourquoi n'avoir dessiné qu'une patte, et non deux? Par ailleurs, y a-t-il un lien entre les ailes des figures animales et celles de l'ange? Dans l'état actuel de la recherche, ces questions restent sans réponse.

En conclusion, si l'on accepte l'interprétation de la scène des deux personnages comme l'illustration du passage de l'Évangile selon Matthieu, 1, 19-20, on peut en déduire qu'il s'agit à ce jour, non seulement de la seule représentation de cette scène sur ostracon (et même sur papyrus), mais, bien que la datation d'un ostracon et d'une main scolaire soient toujours difficiles à établir avec précision, probablement aussi d'une des plus anciennes.

Aspirant du F.R.S. - FNRS

Nathan CARLIG

Université de Liège

Centre de Documentation de Papyrologie Littéraire (CEDOPAL)

der spätantike und des frühen Mittelalters ( $3^{\mathrm{e}}$ éd., Mayence, 1976), pp. 83-44 ( $\left.\mathrm{n}^{\circ} 118\right)$ et pl. 62 et P. Williamson, Medieval Ivory Carvings. Early Christian to Romanesque (Londres, 2010), pp. 156-159 (avec planches). Ces ivoires sont également répertoriés sous le nom de «pixyde de Werden» dans H. LECLERCQ, «Joseph (Saint)», DACL VII, 2 (1927), col. 2663 et ID., «Ivoires», ibid., col. 1976 ( $\left.\mathrm{n}^{\circ} 168\right)$.

(34) Voir ID., «Joseph (Saint)», DACL VII, 2 (1927), col. 2663 et ID., «Âne», op. cit. I, 2 (1907), col. 2059-2060 et fig. 600 et T. KLAUSER, op. cit., col. 279-280 (n 94d) et 295 $\left(\mathrm{n}^{\circ} 15\right)$. Pour une description détaillée, consulter W.F. VOLBACH, op. cit., pp. 93-94 $\left(\mathrm{n}^{\circ} 140\right)$ et pl. 72-74, spéc. 74 B pour le songe de Joseph.

(35) G.J.M. Van LoOn \& A. Delattre, op. cit., p. 126, n. 50. 